\title{
Darrieus Wind Turbine Performance Prediction: Computational Modeling
}

\author{
N.C. Batista ${ }^{1}$, R. Melício ${ }^{1}$, V.M.F. Mendes ${ }^{2}$, J. Figueiredo ${ }^{1}$, and A.H. Reis ${ }^{1}$ \\ ${ }^{1}$ University of Évora, Évora, Portugal \\ nelson.batista@gmail.com, \{rmelicio,jf,ahr\}@uevora.pt \\ ${ }^{2}$ Instituto Superior de Engenharia de Lisboa, Lisbon, Portugal \\ vfmendes@deea.isel.pt
}

\begin{abstract}
The Vertical Axis Wind Turbines Darrieus is facing a rapid installations growth due to the interest for a decentralizing generation. The Darrieus aerodynamic behavior analysis is a hard computational task. This paper starts by offering an insight into the Darrieus wind turbines performance prediction and proposes an algorithm based on the Multiple Streamtube modeling as a prediction approach in order to have an admissible handing in an Internet of things environment.
\end{abstract}

Keywords: Darrieus, Multiple Streamtube model, performance prediction.

\section{Introduction}

The eolic energy source is one of the most cost effective of renewable sources. Electric demand has been satisfied by mixed energy sources and in nowadays the eolic energy source is one of those sources in current industrial use. Investments in eolic energy exploitation are reported as increasing ones [1]. Studies have been conducted [2], [3] regarding the use of eolic energy in order to research behaviors and to justify the approval on the usage of eolic energy in the market, offering tools to help and ease the enterprise R\&D. Also, the eolic energy source is acknowledged as a favorable energy sources for scenarios regarding urban areas [4]. Where, the Vertical Axis Wind Turbine (VAWT) has advantages over the Horizontal Axis Wind Turbines (HAWT) [5], [6]. The high penetration of eolic energy is increasingly affecting the overall performance of the electric grid, due to characteristics of intermittency and high variability in space and time. One of the concerns related to the high penetration of wind turbines is the impact on the system stability and power quality [7]. Hence, the accurate performance prediction of scattered urban wind turbines is crucial in a Smart Grid (SG) due to the fact that a fast and flexible adjustment to the requirements of all players especially the consumer needs of energy usage is an important issue in the SG. The performance prediction of VAWT wind turbines especially in urban areas is a complex task. The Darrieus type VAWT performance prediction is usually a time consuming task, requiring high processing times. The Darrieus VAWT performance prediction can reside in cloud services [8] or be predefined in the wind turbine 
instrumentation in a SG connected to a data network with the possibility for centralized data processing capabilities offered by cloud infrastructures.

The pursuit for the most desirable design for the urban Darrieus VAWT performance prediction in a SG and the relation to the involved data interexchange network on an Internet of Things (IoT) motivated the work presented in this paper. The presented computation algorithm brings the ability to fast predict the Darrieus VAWT aerodynamic performance, contributing to make possible the implementation of services for the interaction between assets and urban wind turbines.

The rest of the paper is organized as follows: Section 2 presents the contribution to innovation: the ability to fast predict the Darrieus VAWT aerodynamic performance, allowing the design of sophisticated services to be implemented on the SG in an IoT environment. Section 3 presents features about models for the Darrieus aerodynamic performance. Section 4 presents the formulation main assumptions for the prediction algorithm. Section 5 presents results from the proposed algorithm and anticipates further work. Finally, concluding remarks are given in Section 6.

\section{Relationship to Internet of Things}

The expected way to interconnect all the assets, players and services in a SG is over the internet infrastructure. Hence, the urban Darrieus VAWT will be an object in the IoT [9] involved in the SG operation [10], [11]. This object requires an adequate modeling to be conveniently processed as a thing in IoT.

This paper computational model is a contribution offering the ability to fast predict the Darrieus VAWT aerodynamic performance, allowing the design of sophisticated services to be implemented on the SG [12]. The computational model can be implemented over an infrastructure interacting not only with the SG but with other services offered over the IoT.

\section{Darrieus Aerodynamic Models}

Despite the complexity of the Darrieus VAWT aerodynamic behavior, mathematical models have been developed [13], [14] in order to predict the performance. These mathematical models have strengths and weaknesses with a more or less accurate prediction, depending on the wind turbine configuration, wind behavior and prediction time consumed. The most commonly used models can be divided in three categories: Vortex model, Cascade model and Blade Element Momentum (BEM) model.

The Vortex model is characterized by: the vortices in the wake of the blades are calculated; the vortex filaments substitute the blades and have the strengths determined by the blade profile coefficients, relative flow and angle of attack. The Vortex model has a good accuracy. But as a main disadvantage, this model requires a high computational time.

The Cascade model arranges the blades in vanes called cascade, placed in equal interspaces. The blades aerodynamic behavior are calculated independently, taking in consideration the angle of attack, the position in the upwind and downwind side of the rotor and the local Reynolds number. Although no convergence problems are reported 
and good predictions results are presented, the cascade model takes a high computational time.

The BEM model is divided in three submodels: Single Streamtube, Multiple Streamtube and Double-Multiple Streamtube.

The BEM modeling combines momentum theory with blade element theory in order to study the behavior of the air flow on the blades and related forces. The turbine in the Single Streamtube model is placed inside a single streamtube and the blades revolution is translated in an actuator disk. The effects outside the streamtube are assumed negligible and the wind speed in the upstream and downstream sides of the turbine are considered constant. This model as a consequence of all the assumptions made does not deliver good prediction accuracy, giving in most cases higher estimate values, but has a fast processing time. The Multiple Streamtube modeling is a variation of the Single Streamtube modeling, dividing the single streamtube in several parallel adjacent streamtubes that are independent from each other and have own undisrupted, wake and induced velocities. Although not accurate, the predicted performance is close to field test values, tending to give values a little higher for high Tip Speed Ratios (TSR) and having a fast processing time. The Double Multiple Streamtube (DMS) modeling is a variation of the Multiple Streamtube modeling, dividing the actuator disc is in two half cycles in tandem, representing the upstream and downstream sides of the rotor. This model presented by Paraschivoiu [15] has suffered several improvements over time and offers a good performance prediction, but presents convergence problems for high solidity turbines, giving high power prediction for high TSR and having a high processing time.

\section{Prediction Algorithm}

The most suitable Darrieus wind turbine performance prediction modeling is the Multiple Streamtube modeling in a SG environment with the assets connected over an internet of thing. This is due to simplicity, reasonable accuracy and low computational processing time, permiting the real time interexchange of data between several SG assets and the wind turbine over an internet of things platform.

The Darrieus wind turbine performance computation divides the wind turbine space horizontally and vertically in several parallel and adjacent streamtubes considered to be independent from each other, having own wake and induced velocities. The streamtube width is given by $\sin (\theta)$, with $c$ the chord of the blade profile and $\theta$ the blade azimuthal angle. The streamtube width when $\theta$ is equal to $0^{\circ}$ and $180^{\circ}$ is considered to be equal to the blade profile height. A variation in the blade azimuth angle $\Delta \theta$ is chosen for the horizontal streamtube division. The number of Horizontal Streamtubes (HST) is equal to $180^{\circ} / \Delta \theta$.

The streamtube height $h$ is equal to the wind turbine height $H$ divided by the number of predefined vertical divisions, VertDiv. Each set of horizontal streamtubes has the same undisrupted wind velocity $V_{\infty}$ that varies with the height. Several forces must be considered in order to study the blade behavior in each streamtube [13], [15]. These forces are computed in function of the airfoil velocities. The airfoil velocities diagram is shown in Fig. 1. 


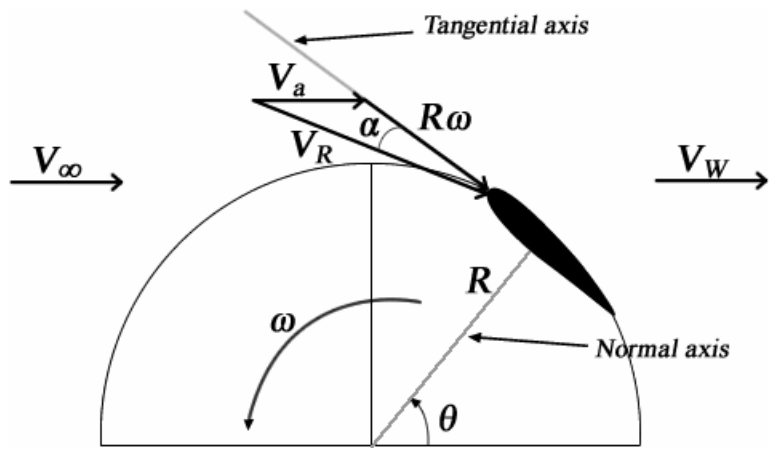

Fig. 1. Airfoil velocities diagram

The relative velocity $V_{R}$ at the blade is given by:

$$
V_{R}=\sqrt{\left(V_{a} \cos \theta+\omega R\right)^{2}+\left(V_{a} \sin \theta\right)^{2}} .
$$

$\omega$ is the rotor angular speed, $R$ is the radius of the wind turbine and $V_{a}$ is the local wind speed at rotor. The Froude's Momentum theory for an actuator disk [15] gives a relation between the undisruptive wind velocity $V_{\infty}$ and the wind speed at the rotor. This relation is given by an induction factor $U$ given by:

$$
V_{a}=V_{\infty}(1-U) \text {. }
$$

Normalizing the relative velocity $V_{R}$ by $V_{\infty},(1)$ is given by:

$$
V_{R} / V_{\infty}=\sqrt{\left(V_{a} / V_{\infty} \cos \theta+\omega R / V_{\infty}\right)^{2}+\left(V_{a} / V_{\infty} \sin \theta\right)^{2}} .
$$

Introducing (2) into (3) and considering $\lambda$ as the TSR, (3) is given by:

$$
V_{R} / V_{\infty}=\sqrt{[(1-U) \cos \theta+\lambda]^{2}+[(1-U) \sin \theta]^{2}} .
$$

The blade angle of attach $\alpha$ is given by:

$$
\alpha=\tan ^{-1}\left(\frac{V_{a} \sin \theta}{V_{a} \cos \theta+\omega R}\right)=\tan ^{-1}\left[\frac{(1-U) \sin \theta}{(1-U) \cos \theta+\lambda}\right] .
$$

The Tangential coefficient $C_{T}$ and normal coefficient $C_{N}$ [13] are given by:

$$
\begin{aligned}
& C_{T}=C_{L} \sin \alpha-C_{D} \cos \alpha \\
& C_{N}=C_{L} \cos \alpha+C_{D} \sin \alpha
\end{aligned}
$$

$C_{L}$ and $C_{D}$ are the lift and drag coefficients of the airfoil for the angle of attack $\alpha$. The aerodynamic trust coefficient $C_{A T}$ is given by: 


$$
C_{A T}=\frac{2}{\pi}\left(\frac{N c}{D}\right)\left(C_{N}-\frac{C_{T}}{\tan \theta}\right)\left(\frac{V_{R}}{V_{\infty}}\right)^{2} .
$$

$N$ is the number of blades and $D$ the diameter of the wind turbine. Applying the momentum theory, the momentum loss trust coefficient $C_{M L T}$ is given by [15]:

$$
C_{M L T}=4 U(1-U) \text {. }
$$

A Multiple Streamtube model diagram is shown in Fig. 2.

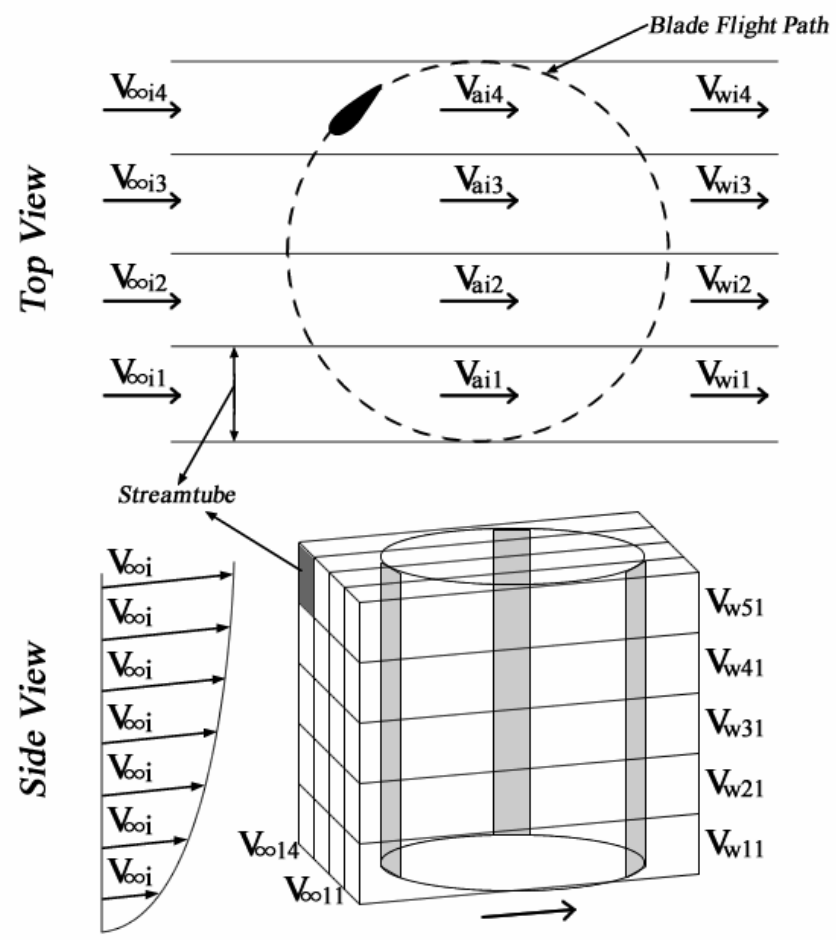

Fig. 2. Multiple Streamtube model diagram

The aerodynamic behavior of the wind turbine in all the streamtubes is calculated in order to compute the Darrieus type VAWT wind turbine performance prediction. A starting interference factor $U$ is chosen for each streamtube. The local wind speed $\mathrm{Va}$, relative wind speed VR, local angle of attack $\alpha$ and Reynolds number are calculated. Hence, the aerodynamic trust coefficient CAT can be calculating, using lift and drag coefficients that can be previously calculated for the blade airfoil and saved in a file or database or calculated in real-time.

The momentum loss trust coefficient $C_{M L T}$ is calculated using the existing interference factor $U$. Both $C_{A T}$ and $C_{M L T}$ are compared and a difference between the two trust coefficients is an error. If this error is outside an error tolerance, a new 
interference factor $U$ is chosen and all the process starts again, until the error between the $C_{A T}$ and $C_{M L T}$ are within the error tolerance. The Multiple Streamtube computational model flowchart is shown in Fig. 3.

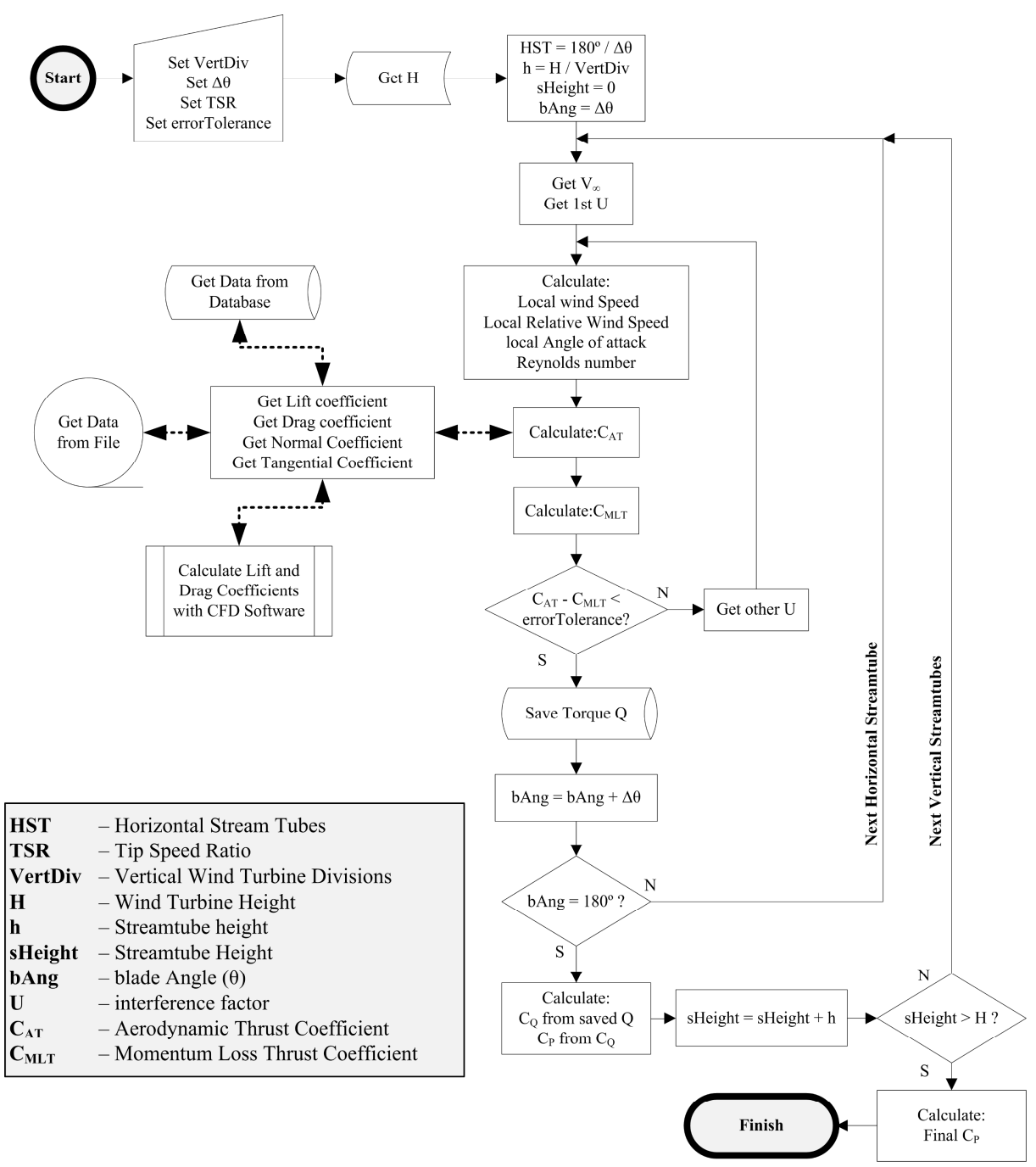

Fig. 3. Multiple Streamtube computational model flowchart

Once an interference factor for a streamtube is found, the torque $Q$ is then calculated and saved.

The final torque coefficient $C_{Q}$ is calculated when all the horizontal and vertical streamtubes are processed. The final torque coefficient $C_{Q}$ is computed by the expression given by: 


$$
C_{Q}=\left(\frac{N c}{D}\right) \frac{\sum_{i=1}^{s}\left[\left(\frac{V_{R}}{V_{\infty}}\right)^{2} C_{T}\right]}{s} .
$$

The final Power coefficient $C_{P}$ is given in function of $C_{Q}(9)$ and the TSR by the expression given by:

$$
C_{P}=C_{Q} \lambda .
$$

The incorporation of the wind turbines behavior in an internet of things platform, requiring fast interexchange of data, is simplified by using this model to compute the aerodynamic performance prediction of Darrieus VAWT.

\section{$5 \quad$ Results and Further Work}

The aerodynamic behaviors of the Darrieus VAWT blade profiles are found to be the most time consuming task. The lift and drag coefficients of the Darrieus VAWT blades can be analyzed in field tests or computed with Computational Fluid Dynamic (CFD) software.

The lift and drag coefficients can be calculated on demand with CFD software, with the disadvantage of increasing the computational time spent in the Darrieus VAWT performance prediction. Hence the use of previously saved data in file or database format is advisable, decreasing the time consumption performance prediction to a few seconds.

The Darrieus VAWT performance prediction computation of the previously presented algorithm developed in ABAP programing language, running on a server with Windows 2008 R2 64-bit Operating System server, two $2.53 \mathrm{GHz}$ processors and 8 GB of Random Access Memory (RAM), takes between 1 to 2 seconds of CPU time to compute, if all the airfoil lift and drag coefficients data are previously saved in a Microsoft SQL Server 2008.

The lift and drag coefficients calculated with CFD software can take between 0.1 seconds up to 5 minutes depending on the CFD software, airfoil complexity, airfoil number of points, blade angle of attack, Reynolds number and convergence criteria for the iterations.

A comparison between the several mathematical performance prediction models elaborated by Sandia National Laboratories is shown in Fig. 4 between the Single Streamtube model and the Multiple Streamtube model (DART computer code) [16]; in Fig. 5 between the Multiple Streamtube model and the Double-Multiple Streamtube model [15]. 


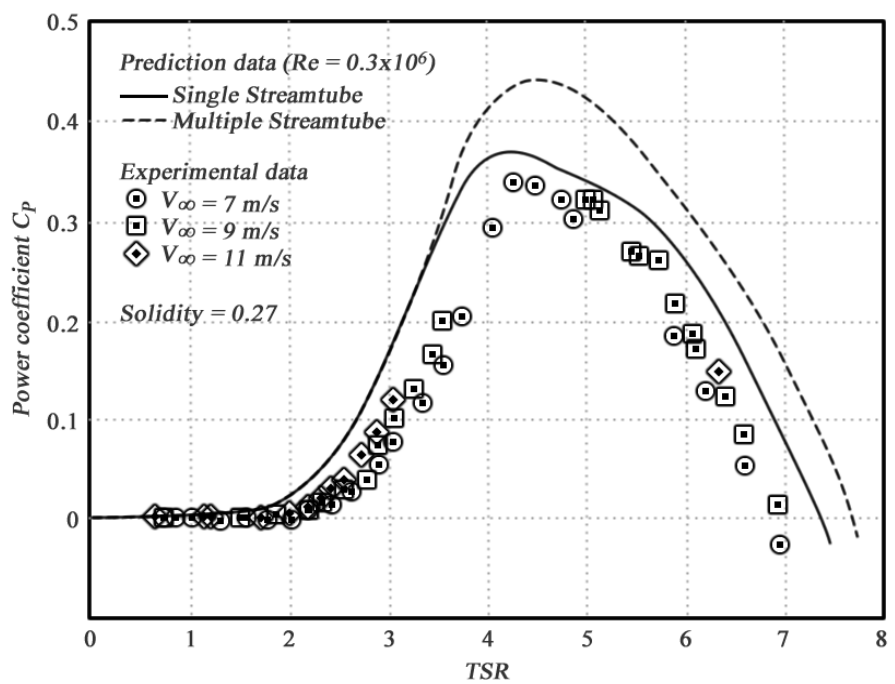

Fig. 4. Single Streamtube model and Multiple Streamtube model comparison with experimental data [16]

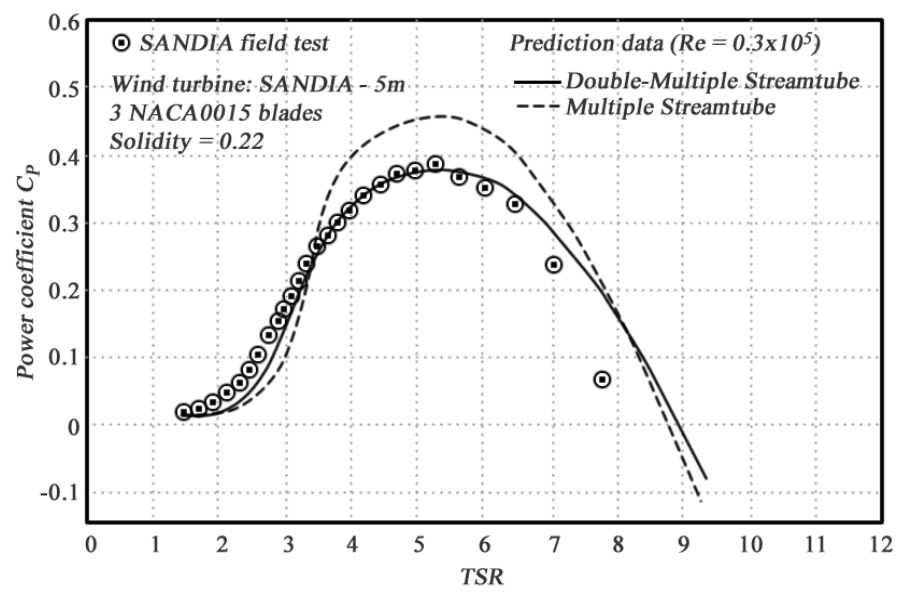

Fig. 5. Multiple Streamtube model and Double-Multiple Streamtube model comparison with experimental data [15]

Although, advantages and disadvantages are intimately related with the Darrieus VAWT design and installation conditions, several most used mathematical performance prediction models advantages and disadvantages are reported. A resume of those reports are presented in [13], and summarized in Table 1. 
Table 1. Darrieus VAWT mathematical performance prediction models advantages and disadvantages [13]

\begin{tabular}{|c|c|c|}
\hline Prediction Model & Advantages & Disadvantages \\
\hline Single Streamtube & $\begin{array}{l}\text { Predicts the overall performance } \\
\text { of a lightly loaded wind turbine. } \\
\text { Very fast computational } \\
\text { prediction. }\end{array}$ & $\begin{array}{l}\text { Does not predict the wind } \\
\text { variations across the rotor } \\
\text { presenting disparities compared } \\
\text { with experimental data. }\end{array}$ \\
\hline Multiple Streamtube & $\begin{array}{l}\text { Predicts the overall performance } \\
\text { reasonably, especially when the } \\
\text { rotor is lightly loaded. } \\
\text { Fast computational prediction. }\end{array}$ & $\begin{array}{l}\text { Some convergence problems, } \\
\text { increasing computational time. } \\
\text { Slight disparities with field } \\
\text { tests, depending on the wind } \\
\text { turbine solidity. }\end{array}$ \\
\hline $\begin{array}{l}\text { Double-Multiple } \\
\text { Streamtube }\end{array}$ & $\begin{array}{l}\text { Offers a good correlation between } \\
\text { the performance prediction and the } \\
\text { experimental data. }\end{array}$ & $\begin{array}{l}\text { Presents convergence problems } \\
\text { that increase computational } \\
\text { time. } \\
\text { Over prediction power for high } \\
\text { solidity wind turbine and higher } \\
\text { TSR. }\end{array}$ \\
\hline Vortex & $\begin{array}{l}\text { With the latest improvements the } \\
\text { model presents a high correlation } \\
\text { between the performance } \\
\text { prediction and the experimental } \\
\text { data. }\end{array}$ & $\begin{array}{l}\text { Takes the highest } \\
\text { computational time of all the } \\
\text { prediction models. }\end{array}$ \\
\hline Cascade & $\begin{array}{l}\text { Reasonable overall prediction for } \\
\text { both low and high solidity wind } \\
\text { turbine. } \\
\text { No convergence problems. }\end{array}$ & $\begin{array}{l}\text { Takes a reasonable computation } \\
\text { time. }\end{array}$ \\
\hline
\end{tabular}

Further work will target the validation with field tests of the presented solution for the Darrieus VAWT performance prediction in the SG included in an IoT. Also, the developments of optimized computational solutions to other Darrieus VAWT performance prediction models are envisaged in the near future.

\section{Conclusions}

Aerodynamic behavior analysis models of Darrieus wind turbines are discussed. The Multiple Streamtube modeling is further analyzed, regarding methodology and formulation. The computation approach is presented in a flowchart and is designed in the most suitable approach for a SG included in an IoT environment, achieving a fast simulation of the aerodynamic performance of a Darrieus VAWT. 


\section{References}

1. Snyder, B., Kaiser, M.J.: A comparison of offshore wind power development in Europe and the U.S.: patterns and drivers of development. Appl. Energy 86(10), 1845-1856 (2009)

2. Melício, R., Mendes, V.M.F., Catalão, J.P.S.: Dynamic stability of wind turbines with permanent magnet machines and power-electronic converters. In: Proc. Power Eng. 2009, Lisbon, Portugal, pp. 484-489 (2009)

3. Faria, D.L., Castro, R., Philippart, C., Gusmão, A.: Wavelets Pre-Filtering in Wind Speed Prediction. In: Proc. Power Eng. 2009, Lisbon, Portugal, pp. 168-173 (2009)

4. Mañana, M.: Small wind energy systems, state of the art and new challenges. In: Proc. ICREPQ 2011, Gran Canaria, Spain (2011)

5. Batista, N.C., Melício, R., Matias, J.C.O., Catalão, J.P.S.: Self-start performance evaluation in Darrieus-type vertical axis wind turbines: methodology and computational tool applied to symmetrical airfoils. In: Proc. ICREPQ 2011, Gran Canaria, Spain (2011)

6. Batista, N.C., Melício, R., Matias, J.C.O., Catalão, J.P.S.: New blade profile for Darrieus wind turbines capable to self-start. In: Proc. IET RPG 2011, Edinburgh, United Kingdom (2011)

7. Melício, R., Mendes, V.M.F., Catalão, J.P.S.: Power converter topologies for wind energy conversion systems: integrated modeling, control strategy and performance simulation. Renewable Energy 35(10), 2165-2174 (2010)

8. Miorandia, D., Sicarib, S., De Pellegrinia, F., Chlamtaca, I.: Internet of things: vision, applications and research challenges. Ad Hoc Networks 10(7), 1497-1516 (2012)

9. Atzoria, L., Ierab, A., Morabitoc, G.: The internet of things: a survey. Computer Networks 54(15), 2787-2805 (2010)

10. Ou, Q., Zhen, Y., Li, X., Zhang, Y., Zeng, L.: Application of internet of things in smart grid power transmission. In: Proc. 3rd FTRA Int. Conf. on Mobile, Ubiquitous, and Int. Comp.- MUSIC, Beijing, China, pp. 96-100 (2012)

11. Bui, N., Castellani, A.P., Casari, P., Zorzi, M.: The internet of energy: a web-enabled smart grid system. IEEE Network 26(4), 39-45 (2012)

12. Atzoria, L., Ierab, A., Morabitoc, M., Nittia, N.: The social internet of things (SIoT) when social networks meet the internet of things: concept, architecture and network characterization. Computer Networks 56(16), 3594-3608 (2012)

13. Islam, M., Ting, D.S.-K., Fartaj, A.: Aerodynamic models for Darrieus-type straightbladed vertical axis wind turbines. Renewable and Sustainable Energy Reviews 12(4), 1087-1109 (2008)

14. Melo, R.R.S., Neto, A.S.: Integral analysis of rotors of a wind generator. Renewable and Sustainable Energy Reviews 16(7), 4809-4817 (2012)

15. Paraschivoiu, I.: Wind Turbine Design: with emphasis on Darrieus concept, 1 st edn. Polytechnic International Press, Canada (2002)

16. Strickland, J.H.: The Darrieus turbine: a performance prediction model using multiple streamtube. Sandia Laboratories Report SAND 75-0431, United States of America (1975) 